\title{
NUMERICAL STUDY OF MACH REFLECTION AND DETONATION BY USING A SECOND-ORDER CE/SE METHOD
}

\author{
Deliang Zhang * \\ LHD, Institute of Mechanics \\ Chinese Academy of Sciences \\ Beijing 100190 \\ S.-M. Liang ** \\ Department of Computer Application Engineering \\ Far East University \\ Tainan County, Taiwan 74448, R.O.C.
}

\begin{abstract}
An improved method of space-time conservation element and solution element (CE/SE) is developed to solve the equations of conservation laws in fluid dynamics. The present method substantially differs in both concept and methodology from the traditional CE/SE method. In this paper the improved second-order CE/SE method is presented in a hexahedral grid. Furthermore, the present CE/SE method was successfully applied to solve the interaction problem of shock waves and detonation. Several numerical examples were also given. Numerical results have compared with the results of experiments and other computational methods. The compared results have shown a good agreement. The improved CE/SE method has higher accuracy and becomes a more prospective scheme.
\end{abstract}

Keywords : Shock wave, Detonation, CE/SE method, Numerical simulation.

\section{INTRODUCTION}

The Space-Time Conservation Element and Solution Element method (CE/SE) originally proposed by Chang [1] is improved to be a high-resolution CFD method for hyperbolic conservation laws. The present CE/SE substantially differs from the traditional wellestablished method. It has some advantages: A unified treatment of space and time; satisfying strictly both local and global flux conservation in space and time; no requirement of operator splitting or alternating direction technology; high resolution for shock capturing. Moreover, it is very general, simpler, and highly efficient for numerical simulation of high-speed fluid flows with shock waves.

For a two-dimensional CE/SE method, different definitions of CEs and SEs may result in different schemes. In original definitions of CE and SE [1], every SE corresponds to three CEs. This definition agrees with the idea of space and time conservation. However, the directions of grid numbering and $x, y$ axes do not coincide with each other, which will cause difficulties in constructing schemes and extension to three dimensions. Liu and $\mathrm{Xu}$ [2], Zhang et al. [3], and Zhang et al. [4] respectively modified the definitions of $\mathrm{SE}$ and CE and proposed a hexahedral definition in which only one CE was required by every SE and the relationships between variables and their partial derivatives were determined by the continuity at the common grid point in the neighborhood of SEs. The present $\mathrm{SE} / \mathrm{CE}$ definition is much more convenient than that in Ref. 4 , because it does not need boundary conditions in the half-time steps.

Up to authors' knowledge, most of CE/SE methods mentioned above have only first order accuracy except for references 5 and 6 . The theory of high order accuracy for the CE/SE method has not been well established. In this paper, the original two-dimensional $\mathrm{CE} / \mathrm{SE}$ structures have been modified and improved to establish a high-order CE/SE method. Thus the highorder $\mathrm{CE} / \mathrm{SE}$ method is applied to some complicated problems of Mach reflections and detonation.

\section{TWO-DIMENSIONAL, SECOND-ORDER CE/SE METHOD}

Consider the two-dimensional conservation equations for a compressible flow with a source term $S$ :

$$
\frac{\partial \mathbf{Q}}{\partial t}+\frac{\partial \mathbf{E}(\mathbf{U})}{\partial x}+\frac{\partial \mathbf{F}(\mathbf{U})}{\partial y}=\mathbf{S}
$$

\footnotetext{
${ }^{*}$ Professor ${ }^{* *}$ Professor, corresponding author
} 
To construct a CE/SE scheme, let $(j, k, n)$ denote the set of space-time mesh points, where $n=0,1 / 2,1$, $3 / 2, \ldots$ denotes the time index, $j=0, \pm 1 / 2, \pm 1, \pm 3 / 2, \ldots$. for the $x$ direction and $k=0, \pm 1 / 2, \pm 1, \pm 3 / 2, \ldots$ for the $y$ direction.

According to the $\mathrm{CE}$ and SE definitions of Zhang et al. [3], the projection of grid points on the $x y$ plane is shown in Fig. 1. Note that in Fig. 1, grid points denoted by " $\bullet$ " and " $\circ$ " are different at the $\Delta t / 2$ time step. For every grid point $P^{\prime}(j, k, n)$, there exists a solution element $\operatorname{SE}\left(P^{\prime}\right)$, referring to Fig. 2, and the corresponding conservation element $\mathrm{CE}\left(P^{\prime}\right)$ is the hexahedron $A C E G A^{\prime} C^{\prime} E^{\prime} G^{\prime}$, as shown in Fig. 3.

Let $x_{1}=x, x_{2}=y, x_{3}=t$ be the coordinates of the Euclidean space $E_{3}$. By using the Gauss' divergence theorem, Eq. (1) can be written by an integral conservation law

$$
\int_{S(V)} \mathbf{H}_{m} \cdot \mathrm{d} \mathbf{s}=0
$$

where $S(V)$ is the boundary of an arbitrary space-time region $V$ in $E_{3}$, and $\mathrm{d} \mathbf{s}=\mathrm{d} \boldsymbol{\sigma} \cdot \mathbf{n}$. Here $\mathrm{d} \boldsymbol{\sigma}$ and $\mathbf{n}$ denote the area and the outward unit normal vector of a surface element on $S(V) ; \mathbf{H}_{m}=\left(Q_{m}, E_{m}, F_{m}\right)$ is the space-time flux vector, where $Q_{m}, E_{m}, F_{m}$ are the components of the vector $(\mathbf{Q}, \mathbf{E}, \mathbf{F})$, respectively; $\mathbf{S}$ is the source term vector of conservation equations with the components $S_{m}$.

For an arbitrary solution element $\mathrm{SE}\left(P^{\prime}\right)$, we suppose that $Q_{m}, E_{m}, F_{m}$ and $S_{m}$ at point $(x, y, t)$ in $\mathrm{SE}\left(P^{\prime}\right)$ can be approximated by the second-order Taylor's expansion near point $P^{\prime}(j, k, n)$, i.e.,

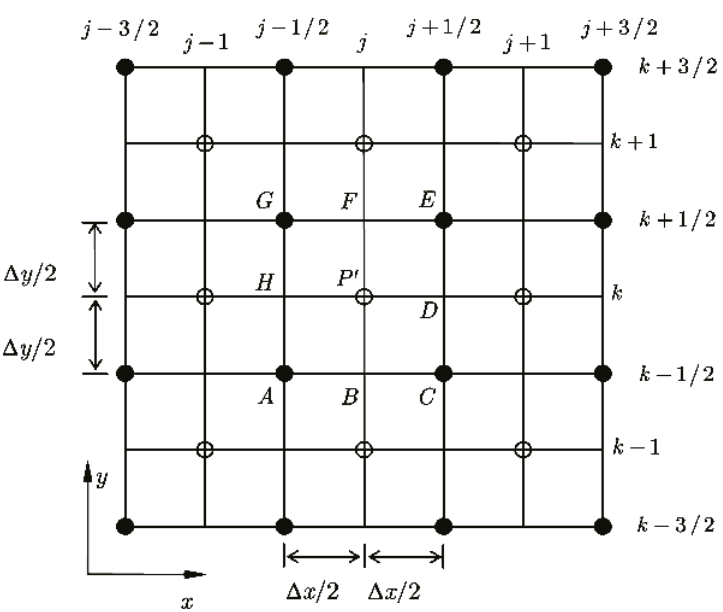

Fig. 1 Grid point projection on the $x y$ plane

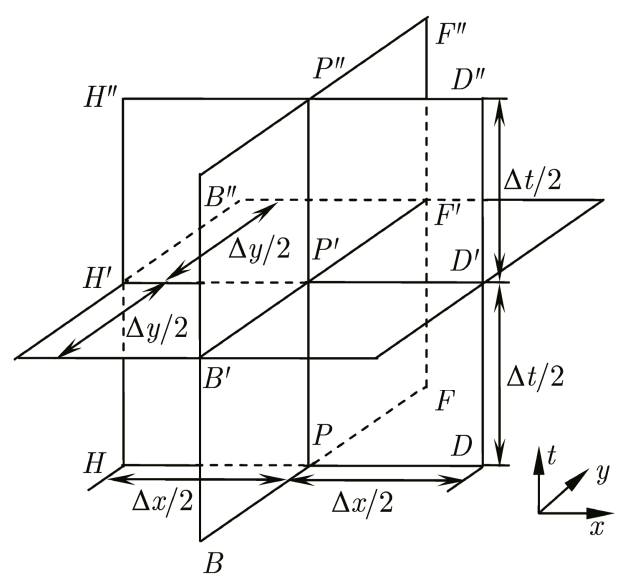

Fig. 2 Solution element $\operatorname{SE}\left(P^{\prime}\right)$

$$
\begin{aligned}
Q_{m}(\delta x, \delta y, \delta t)_{P^{\prime}}= & \left(Q_{m}\right)_{P^{\prime}}+\left(Q_{m x}\right)_{P^{\prime}} \delta x+\left(Q_{m y}\right)_{P^{\prime}} \delta y+\left(Q_{m t}\right)_{P^{\prime}} \delta t \\
& +\frac{1}{2}\left(Q_{m x x}\right)_{P^{\prime}}(\delta x)^{2}+\frac{1}{2}\left(Q_{m y y}\right)_{P^{\prime}}(\delta y)^{2}+\frac{1}{2}\left(Q_{m t t}\right)_{P^{\prime}}(\delta t)^{2} \\
& +\left(Q_{m x y}\right)_{P^{\prime}} \delta x \delta y+\left(Q_{m x t}\right)_{P^{\prime}} \delta x \delta t+\left(Q_{m y t}\right)_{P^{\prime}} \delta y \delta t \\
E_{m}(\delta x, \delta y, \delta t)_{P^{\prime}}= & \left(E_{m}\right)_{P^{\prime}}+\left(E_{m x}\right)_{P^{\prime}} \delta x+\left(E_{m y}\right)_{P^{\prime}} \delta y+\left(E_{m t}\right)_{P^{\prime}} \delta t \\
& +\frac{1}{2}\left(E_{m x x}\right)_{P^{\prime}}(\delta x)^{2}+\frac{1}{2}\left(E_{m y y}\right)_{P^{\prime}}(\delta y)^{2}+\frac{1}{2}\left(E_{m t t}\right)_{P^{\prime}}(\delta t)^{2} \\
& +\left(E_{m x y}\right)_{P^{\prime}} \delta x \delta y+\left(E_{m x t}\right)_{P^{\prime}} \delta x \delta t+\left(E_{m y t}\right)_{P^{\prime}} \delta y \delta t \\
F_{m}(\delta x, \delta y, \delta t)_{P^{\prime}}= & \left(F_{m}\right)_{P^{\prime}}+\left(F_{m x}\right)_{P^{\prime}} \delta x+\left(F_{m y}\right)_{P^{\prime}} \delta y+\left(F_{m t}\right)_{P^{\prime}} \delta t \\
& +\frac{1}{2}\left(F_{m x x}\right)_{P^{\prime}}(\delta x)^{2}+\frac{1}{2}\left(F_{m y y}\right)_{P^{\prime}}(\delta y)^{2}+\frac{1}{2}\left(F_{m t t}\right)_{P^{\prime}}(\delta t)^{2} \\
& +\left(F_{m x y}\right)_{P^{\prime}} \delta x \delta y+\left(F_{m x t}\right)_{P^{\prime}} \delta x \delta t+\left(F_{m y t}\right)_{P^{\prime}} \delta y \delta t
\end{aligned}
$$

where $\mathrm{d} x=x-x_{p^{\prime}}, \mathrm{d} y=y-y_{p^{\prime}}, \mathrm{d} t=t-t_{p^{\prime}} ; x_{p^{\prime}}, y_{p^{\prime}}$ and $t_{p^{\prime}}$ are the position coordinates of space and time for point $P^{\prime}$; $(V)_{P^{\prime}},\left(V_{x}\right)_{P^{\prime}},\left(V_{y}\right)_{P^{\prime}},\left(V_{t}\right)_{P^{\prime}},\left(V_{x x}\right)_{P^{\prime}},\left(V_{y y}\right)_{P^{\prime}},\left(V_{t t}\right)_{P^{\prime}}, \quad\left(V_{x y}\right)_{P^{\prime}},\left(V_{x t}\right)_{P^{\prime}}$ and $\left(V_{y t}\right)_{P^{\prime}}$ are approximately constant values of $V$ and its first-order and second-order derivatives in $x, y$ and $t$ directions for point $P^{\prime}$ respectively, in which $V$ denotes $Q_{m}, E_{m}, F_{m}$. Here the expression for $S_{m}(\delta x, \delta y, \delta t)_{P^{\prime}}$ is similar to those for $Q_{m}, E_{m}, F_{m}$.

Substituting Eq. (3) into Eq. (1) yields 


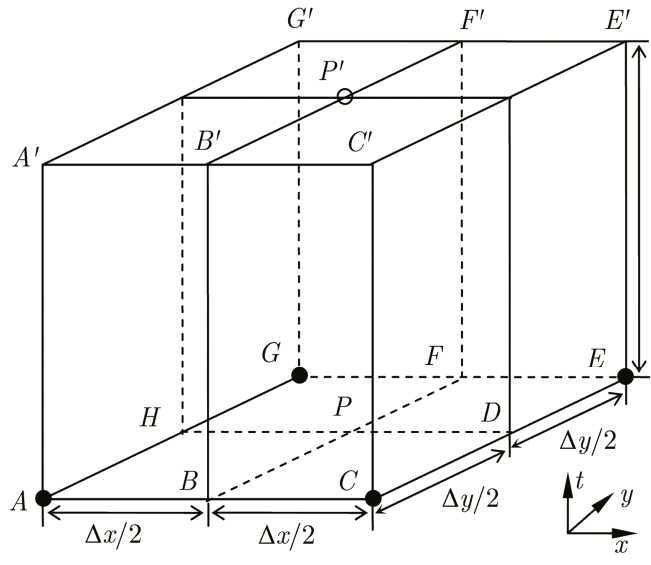

Fig. 3 Conservation element $\mathrm{CE}\left(P^{\prime}\right)$

$$
\begin{aligned}
& \left(Q_{m t}\right)_{P^{\prime}}=-\left(E_{m x}\right)_{P^{\prime}}-\left(F_{m y}\right)_{P^{\prime}} \\
& \left(Q_{m x t}\right)_{P^{\prime}}=-\left(E_{m x x}\right)_{P^{\prime}}-\left(F_{m x y}\right)_{P^{\prime}} \\
& \left(Q_{m y t}\right)_{P^{\prime}}=-\left(E_{m x y}\right)_{P^{\prime}}-\left(F_{m y y}\right)_{P^{\prime}} \\
& \left(Q_{m t t}\right)_{P^{\prime}}=-\left(E_{m x t}\right)_{P^{\prime}}-\left(F_{m y t}\right)_{P^{\prime}}
\end{aligned}
$$

Since $E_{m}$ and $F_{m}$ are functions of $Q_{m}$, the above equations imply that the variables required in computation are $\left(Q_{m}\right)_{P^{\prime}}$ and first- and second-order space derivatives $\left(Q_{m x}\right)_{P^{\prime}},\left(Q_{m y}\right)_{P^{\prime}},\left(Q_{m x x}\right)_{P^{\prime}},\left(Q_{m x y}\right)_{P^{\prime}}$ and $\left(Q_{m y y}\right)_{P^{\prime}}$ at point $P^{\prime}$ respectively.

The conservation element $\mathrm{CE}\left(P^{\prime}\right)$ is demonstrated in Fig. 3. From Fig. 3, it is can be seen that the $\mathrm{CE}\left(P^{\prime}\right)$ is constructed by several solution elements $\mathrm{SE}\left(P^{\prime}\right) \mathrm{s}$ and the known $\mathrm{SEs}$, such as $\mathrm{SE}(A), \mathrm{SE}(C), \mathrm{SE}(E)$ and $\mathrm{SE}(G)$. Assuming that the flux vector satisfies an integral conservation law on every $\mathrm{CE}\left(P^{\prime}\right)$, with the help of Eq. (3), Eq. (2) on the surfaces of $\mathrm{CE}\left(P^{\prime}\right)$ after integration becomes:

$$
\left(Q_{m}\right)_{P^{\prime}}+\frac{\Delta x^{2}}{24}\left(Q_{m x x}\right)_{P^{\prime}}+\frac{\Delta y^{2}}{24}\left(Q_{m y y}\right)_{P^{\prime}}=\frac{1}{4}\left(\bar{Q}+\frac{\Delta t}{\Delta x} \bar{E}+\frac{\Delta t}{\Delta y} \bar{F}\right)
$$

Expanding the functions, $\hat{Q}_{m}(\delta x, \delta y, \delta t)_{P^{\prime}}, \quad \hat{E}_{m}(\delta x$, $\delta y, \delta t)_{P^{\prime}}$, and $\hat{F}_{m}(\delta x, \delta y, \delta t)_{P^{\prime}}$, yields

$\hat{Q}_{m}(\delta x, \delta y, \delta t)_{P^{\prime}}=\left(Q_{m}\right)_{P^{\prime}}+\left(Q_{m x}\right)_{P^{\prime}} \delta x+\left(Q_{m y}\right)_{P^{\prime}} \delta y+\left(Q_{m t}\right)_{P^{\prime}} \delta t$

$$
+\frac{1}{6}\left(Q_{m x x}\right)_{P^{\prime}}(\delta x)^{2}+\frac{1}{6}\left(Q_{m y y}\right)_{P^{\prime}}(\delta y)^{2}+\left(Q_{m x y}\right)_{P^{\prime}} \delta x \delta y
$$

$\hat{E}_{m}(\delta x, \delta y, \delta t)_{P^{\prime}}=\left(E_{m}\right)_{P^{\prime}}+\left(E_{m x}\right)_{P^{\prime}} \delta x+\left(E_{m y}\right)_{P^{\prime}} \delta y+\left(E_{m t}\right)_{P^{\prime}} \delta t$

$$
+\frac{1}{6}\left(E_{m y y}\right)_{P^{\prime}}(\delta y)^{2}+\frac{1}{6}\left(E_{m t t}\right)_{P^{\prime}}(\delta t)^{2}+\left(E_{m y t}\right)_{P^{\prime}} \delta y \delta t
$$

$\hat{F}_{m}(\delta x, \delta y, \delta t)_{P^{\prime}}=\left(F_{m}\right)_{P^{\prime}}+\left(F_{m x}\right)_{P^{\prime}} \delta x+\left(F_{m y}\right)_{P^{\prime}} \delta y+\left(F_{m t}\right)_{P^{\prime}} \delta t$

$$
+\frac{1}{6}\left(F_{m x x}\right)_{P^{\prime}}(\delta x)^{2}+\frac{1}{6}\left(F_{m t t}\right)_{P^{\prime}}(\delta t)^{2}+\left(F_{m x t}\right)_{P^{\prime}} \delta x \delta t
$$

So the $\bar{Q}, \bar{E}$ and $\bar{F}$ in Eq. (5) can be expressed as

$$
\begin{aligned}
& \bar{Q}=\hat{Q}_{m}\left(\frac{\Delta x}{4}, \frac{\Delta y}{4}, 0\right)_{A}+\hat{Q}_{m}\left(-\frac{\Delta x}{4}, \frac{\Delta y}{4}, 0\right)_{C}+\hat{Q}_{m}\left(-\frac{\Delta x}{4},-\frac{\Delta y}{4}, 0\right)_{E}+\hat{Q}_{m}\left(\frac{\Delta x}{4},-\frac{\Delta y}{4}, 0\right)_{G} \\
& \bar{E}=\hat{E}_{m}\left(0, \frac{\Delta y}{4}, \frac{\Delta t}{4}\right)_{A}-\hat{E}_{m}\left(0, \frac{\Delta y}{4}, \frac{\Delta t}{4}\right)_{C}-\hat{E}_{m}\left(0,-\frac{\Delta y}{4}, \frac{\Delta t}{4}\right)_{E}+\hat{E}_{m}\left(0,-\frac{\Delta y}{4}, \frac{\Delta t}{4}\right)_{G} \\
& \bar{F}=\hat{F}_{m}\left(\frac{\Delta x}{4}, 0, \frac{\Delta t}{4}\right)_{A}+\hat{F}_{m}\left(-\frac{\Delta x}{4}, 0, \frac{\Delta t}{4}\right)_{C}-\hat{F}_{m}\left(-\frac{\Delta x}{4}, 0, \frac{\Delta t}{4}\right)_{E}-\hat{F}_{m}\left(\frac{\Delta x}{4}, 0, \frac{\Delta t}{4}\right)_{G}
\end{aligned}
$$

From Eq. (5), the spatial second-order derivatives for $\left(Q_{m x x}\right)_{P^{\prime}}$ and $\left(Q_{m y y}\right)_{P^{\prime}}$ at point $P^{\prime}$ must be known firstly for solving $\left(Q_{m}\right)_{P^{\prime}}$. With the estimated value in $\operatorname{SE}\left(P^{\prime}\right)$, approximated from the previous half-time step, the second-order derivatives for $\left(Q_{m x x}\right)_{P^{\prime}}$ and $\left(Q_{m y y}\right)_{P^{\prime}}$ at point $P^{\prime}$ can be expressed as

$$
\begin{aligned}
& \left(Q_{m x x}\right)_{P^{\prime}}=\frac{\left(\hat{Q}_{m x}\right)_{C}-\left(\hat{Q}_{m x}\right)_{A}+\left(\hat{Q}_{m x}\right)_{E}-\left(\hat{Q}_{m x}\right)_{G}}{2 \Delta x} \\
& \left(\hat{Q}_{m x}\right)=\left(Q_{m x}\right)+\frac{\Delta t}{2}\left(Q_{m x t}\right)
\end{aligned}
$$




$$
\begin{aligned}
& \left(Q_{m y y}\right)_{P^{\prime}}=\frac{\left(\hat{Q}_{m y}\right)_{C}-\left(\hat{Q}_{m y}\right)_{A}+\left(\hat{Q}_{m y}\right)_{E}-\left(\hat{Q}_{m y}\right)_{G}}{2 \Delta y} \\
& \left(\hat{Q}_{m y}\right)=\left(Q_{m y}\right)+\frac{\Delta t}{2}\left(Q_{m y t}\right)
\end{aligned}
$$

For the cross derivatives, we have

$$
\left(Q_{m x y}\right)_{P^{\prime}}=\left(Q_{m y x}\right)_{P^{\prime}}=\frac{\left(Q_{m x y}\right)_{P^{\prime}}^{\prime}+\left(Q_{m y x}\right)_{P^{\prime}}^{\prime}}{2}
$$

where

$$
\begin{aligned}
& \left(Q_{m x y}\right)_{P^{\prime}}^{\prime}=\frac{\left(\hat{Q}_{m x}\right)_{C}-\left(\hat{Q}_{m x}\right)_{A}+\left(\hat{Q}_{m x}\right)_{E}-\left(\hat{Q}_{m x}\right)_{G}}{2 \Delta y} \\
& \left(Q_{m y x}\right)_{P^{\prime}}^{\prime}=\frac{\left(\hat{Q}_{m y}\right)_{C}-\left(\hat{Q}_{m y}\right)_{A}+\left(\hat{Q}_{m y}\right)_{E}-\left(\hat{Q}_{m y}\right)_{G}}{2 \Delta x}
\end{aligned}
$$

Using the continuous condition at points $A^{\prime}, C^{\prime}, E^{\prime}$, and $G^{\prime}$, the left and right derivatives of $Q_{m}$ at the $x$ and $y$ directions can be expressed as

$$
\begin{aligned}
& \left(Q_{m x}\right)_{P^{\prime}}^{-}=-\frac{1}{\Delta x}\left[Q_{m}\left(0,0, \frac{\Delta t}{2}\right)_{A}+Q_{m}\left(0,0, \frac{\Delta t}{2}\right)_{G}-2\left(Q_{m}\right)_{P^{\prime}}\right] \\
& \left(Q_{m x}\right)_{P^{\prime}}^{+}=+\frac{1}{\Delta x}\left[Q_{m}\left(0,0, \frac{\Delta t}{2}\right)_{C}+Q_{m}\left(0,0, \frac{\Delta t}{2}\right)_{E}-2\left(Q_{m}\right)_{P^{\prime}}\right] \\
& \left(Q_{m y}\right)_{P^{\prime}}^{-}=-\frac{1}{\Delta y}\left[Q_{m}\left(0,0, \frac{\Delta t}{2}\right)_{A}+Q_{m}\left(0,0, \frac{\Delta t}{2}\right)_{C}-2\left(Q_{m}\right)_{P^{\prime}}\right] \\
& \left(Q_{m y}\right)_{P^{\prime}}^{+}=+\frac{1}{\Delta y}\left[Q_{m}\left(0,0, \frac{\Delta t}{2}\right)_{E}+Q_{m}\left(0,0, \frac{\Delta t}{2}\right)_{G}-2\left(Q_{m}\right)_{P^{\prime}}\right]
\end{aligned}
$$

To avoid numerical instability in the case of discontinuity, the derivatives in the expressions (8), (9) and (10) are written in form of weighted average

$$
\begin{aligned}
& \left(Q_{m x}\right)_{P^{\prime}}=W\left[\left(Q_{m x}\right)_{P^{\prime}}^{-},\left(Q_{m x}\right)_{P^{\prime}}^{+}, \alpha\right] \\
& \left(Q_{m y}\right)_{P^{\prime}}=W\left[\left(Q_{m y}\right)_{P^{\prime}}^{-},\left(Q_{m y}\right)_{P^{\prime}}^{+}, \alpha\right]
\end{aligned}
$$

where $\alpha$ is an adjustable constant and usually was chosen to equal to $1 \sim 2$. The weighted equation $W$ is expressed as

$$
W\left[x_{-}, x_{+}, \alpha\right]=\frac{\left|x_{+}\right|^{\alpha} x_{-}+\left|x_{-}\right|^{\alpha} x_{+}}{\left|x_{+}\right|^{\alpha}+\left|x_{-}\right|^{\alpha}}
$$

Note that, expression (13) is obviously the same as expressions (8), (9) and (10) in the case of $\alpha=0$.

\section{VERIFICATION OF THE IMPROVED CE/SE SCHEME}

For numerical simulation of the problem of shockwave reflection, we adopted an ideal gas model and no source term $(\mathbf{S}=0)$ in the governing equation (1). Consequently the two-dimensional Euler conservation equations become

$$
\frac{\partial \mathbf{Q}}{\partial t}+\frac{\partial \mathbf{E}(\mathbf{U})}{\partial x}+\frac{\partial \mathbf{F}(\mathbf{U})}{\partial y}=0
$$

In order to check the numerical accuracy of the developed second-order $\mathrm{CE} / \mathrm{SE}$ scheme, the problem of steady-state shock reflection was simulated by using four different schemes. They were the first- and second-order CE/SE schemes, a second-order MUSCL scheme, and a first-order Roe scheme. The problem has the same flow conditions in Ref. 7.

Figure 4 shows the distribution of dimensionless pressure $c_{p}=p / p_{\infty}$ at $y=0.5$. One can see that the accuracy of the first-order CE/SE scheme is higher than that for the first-order Roe scheme and for the second-order MUSCL scheme. Clearly, the second-order $\mathrm{CE} / \mathrm{SE}$ scheme is more accurate than the first-order $\mathrm{CE} / \mathrm{SE}$ scheme.

\section{REFLECTION AND DEFLECTION OF SHOCK WAVE}

\subsection{Four Mach Reflections of Shock Wave on a Wedge}

When a shock wave impinges a wedge, four wellknown shock wave reflections occur: Regular reflection (RR), single-Mach (SMR), complex-Mach (CMR) and double-Mach (DMR) reflections [8]. Figure 5 are numerical results obtained by using the second-order $\mathrm{CE} / \mathrm{SE}$ method. One can see that shock wave configurations of RR, SMR, CMR and DMR are well simulated. Note that the problem was computed on a Cartesian coordinate system. A linear interpolation method was used for flow property on the grid points on the wall. The boundary condition on the wall is the slip condition that is the tangency condition with a zero value of the normal velocity component on the wall. The accuracy of the present method may be degraded at the wall. However, the computed wave configurations as shown in Fig. 5 are well predicted.

\subsection{Interaction of Shock Wave with an Obstacle}

Two cases of shock wave interacting with an obstacle (a backward-facing step) in a channel are simulated first. Figure 6 shows numerical and experimental results for shock wave with incident shock Mach numbers of $M s=1.3$ and 3.0. From Fig. 6, good agreement is obtained by comparing numerical isopycnics with experimental shadowgraphs except for the vortex shock. Further gird refinement may be needed.

Next, the problem of a shock wave with $M s=3.0$ passing through a tunnel with aligned baffles is considered. Numerical and experimental results are shown in Fig. 7. When a shock wave entered into the tunnel, it reflected from the first baffle, resulting in a couple of vortex occurring behind the baffle. Later, reflection, diffraction and interaction of reflected shock waves with vortices occurred. From the present numerical results, we can conclude that our second-order $\mathrm{CE} / \mathrm{SE}$ scheme with a new structure of CEs and SEs did enhance computational accuracy. 


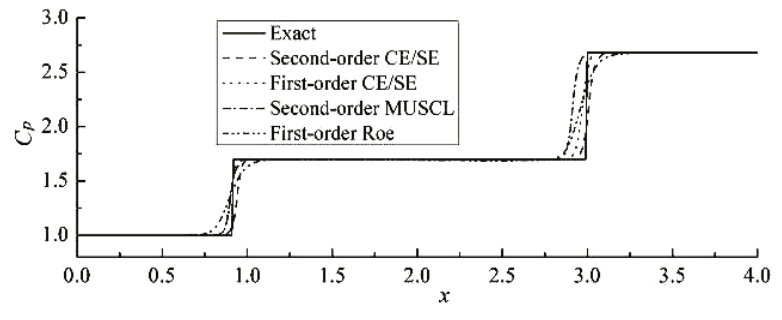

Fig. 4 Pressure coefficient at $y=0.5$ for the steadystate shock reflection problem $(400 \times 100$ meshes)

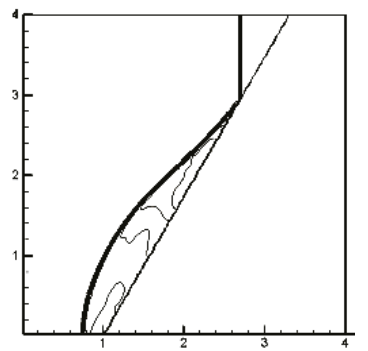

(a) Regular reflection

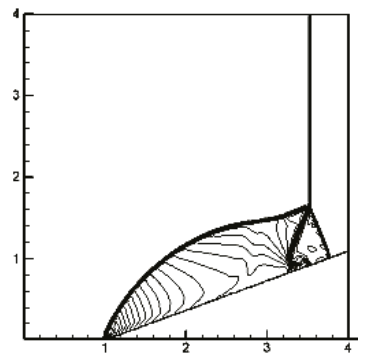

(c) Complex-Mach reflection

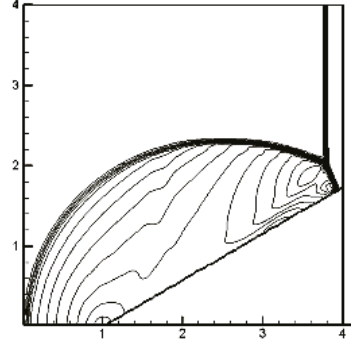

(b) Single-Mach reflection

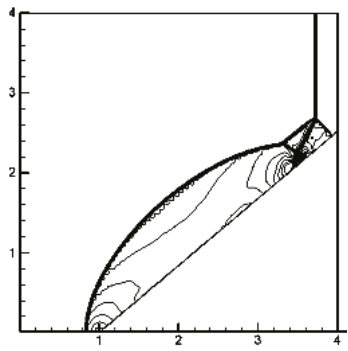

(d) Double-Mach reflection
Fig. 5 Four types of shock wave reflection configuration
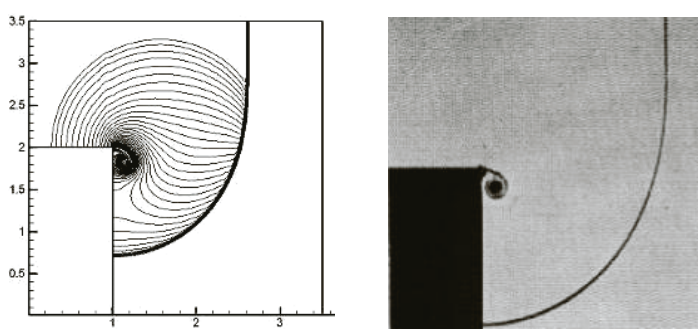

(a) $M s=1.3$
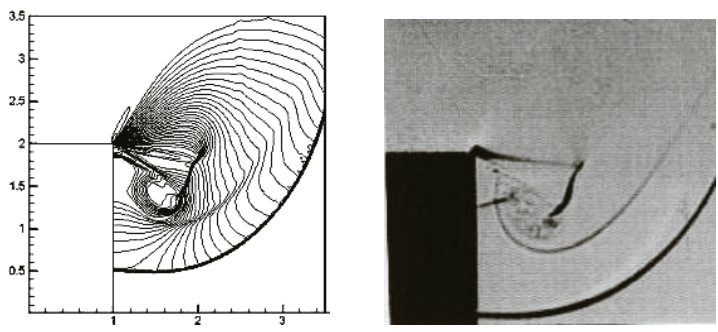

(b) $M s=3.0$

Fig. 6 Numerical and experimental results of shock wave passing a backward step in a channel $(400 \times 400$ meshes $)$
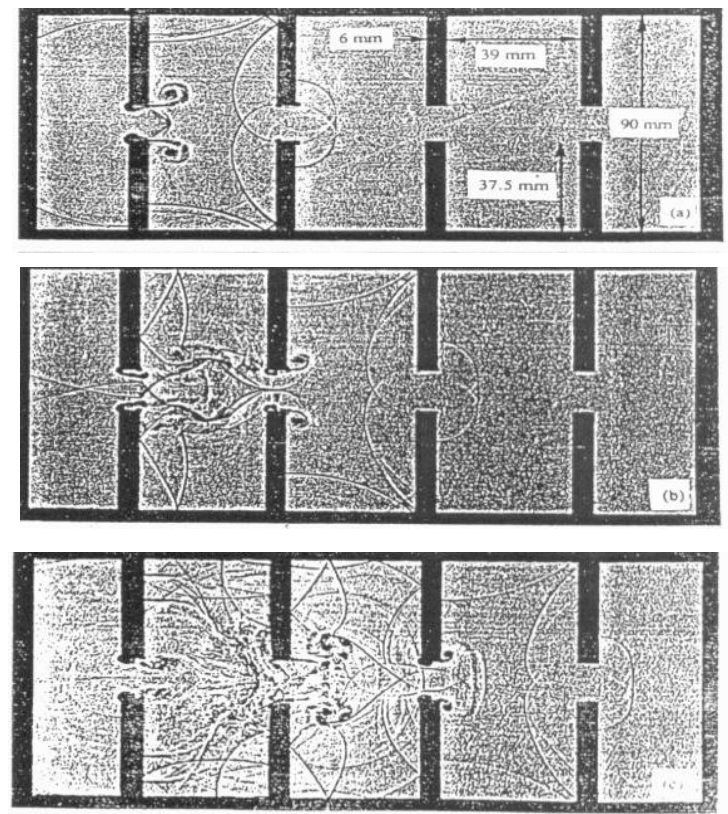

(a) shadow cinematography
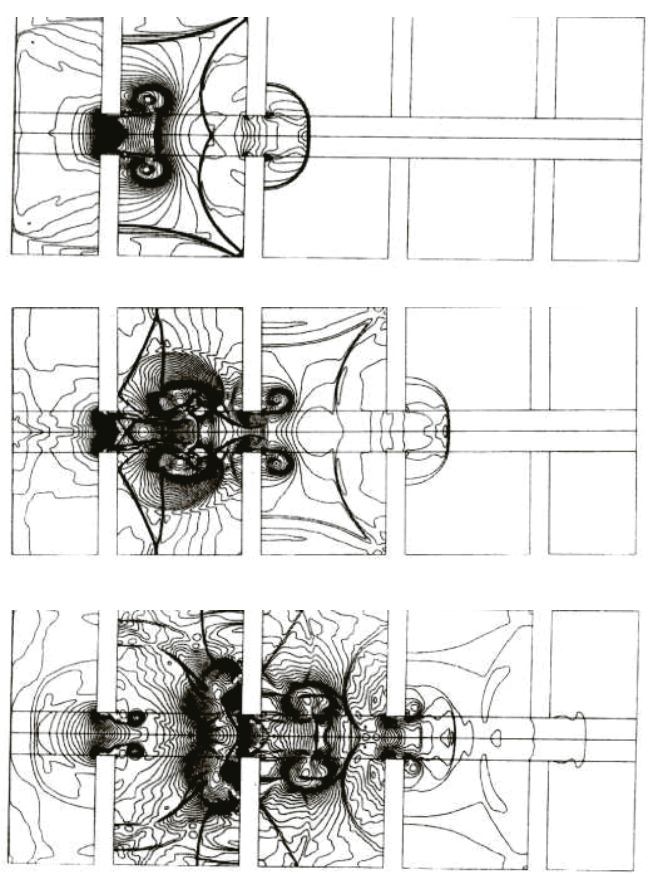

(b) computational results

Fig. 7 Numerical and experimental results of a shock wave passing through a channel with aligned baffles

\section{MACH REFLECTION OF GASEOUS DETONATION}

When a gaseous detonation wave reflects on a wedge, Mach Reflection of a gaseous detonation wave has occurred. Mach Reflection of a gaseous detonation wave on a wedge will form cellular structures (cellular grid with half-diamond shape) in flow field. It is known that the size and shape of cellular structures before and after Mach reflection are marked changed and they are relative to wedge angle and initial condition of gaseous mixture [9]. 


\subsection{Governing Equations of Gaseous Detonation}

The governing equations for a chemically reacting gas mixture problem are the two- dimensional Euler conservation equations with a source term $\mathbf{S}$ :

$$
\begin{gathered}
\frac{\partial \mathbf{Q}}{\partial t}+\frac{\partial \mathbf{E}}{\partial x}+\frac{\partial \mathbf{F}}{\partial y}=\mathbf{S} \\
\mathrm{Q}=\left(\begin{array}{c}
\rho C_{1} \\
\rho C_{2} \\
\vdots \\
\rho C_{n s} \\
m \\
n \\
E
\end{array}\right), \quad \mathrm{E}=\left(\begin{array}{c}
m C_{1} \\
m C_{2} \\
\vdots \\
m C_{n s} \\
m^{2} / \rho+p \\
m n / \rho \\
(E+p) m / \rho
\end{array}\right), \\
\left.\mathrm{F}=\left(\begin{array}{c}
\dot{\omega}_{1} \\
\dot{\omega}_{2} \\
\vdots \\
\vdots \\
n C_{n s} \\
m n / \rho \\
n^{2} / \rho+p \\
(E+p) n / \rho
\end{array}\right), \quad \begin{array}{c}
\dot{\omega}_{n s} \\
0 \\
0 \\
0
\end{array}\right)
\end{gathered}
$$

and $E=e+\rho / 2\left(u^{2}+v^{2}\right)$. The source term $\mathbf{S}$ in eq. (16) describes the chemical reaction. The treatment of the source term is to obtain its value at the current time from the previous variable value by time linearization. In this way, computational efficiency was found to be much improved unlike the method of Newton's iteration. The detail can be found in reference 10 .

\subsection{Chemical Reaction Model}

In numerical simulation three chemical models were usually adopted: The C-J model, the simplified two-step chemical model and the detailed chemically reacting model. The detailed chemically reacting model can truly describe a chemical reaction process. Therefore we adopted a detailed chemically reacting model with eight species $\left(\mathrm{H}_{2}, \mathrm{O}_{2}, \mathrm{H}, \mathrm{O}, \mathrm{HO}, \mathrm{HO}_{2}, \mathrm{H}_{2} \mathrm{O}, \mathrm{H}_{2} \mathrm{O}_{2}\right)$, i.e., twenty-reaction mechanism to simulate hydrogenoxygen detonation process. Table 1 shows the detailed chemical reaction model of a hydrogen-oxygen mixture and the corresponding parameters [11].

\subsection{Verification of 1-D Gaseous Detonation Process}

A stoichiometric $\mathrm{H}_{2}-\mathrm{O}_{2}$ mixture $(\phi=1.0)$ was filled in a channel with a length of $0.04 \mathrm{~m}$, in which initial pressure and temperature were $1 \mathrm{~atm}$ and $298 \mathrm{~K}$, respectively. There is an igniting zone with $0.2 \mathrm{~mm}$ width in the left end of the channel. The igniting zone has higher initial pressure and temperature, i.e., 28atm and $3874 \mathrm{~K}$, respectively. After ignition a detonation wave propagates from the left to right in the channel.

Figure 8 shows the pressure and temperature of developing process of detonation wave propagation.
Table 1 Detailed chemical reaction model of a hy-

\begin{tabular}{|c|c|c|c|c|c|c|c|c|c|}
\hline & Reaction & $A$ & $n$ & $E a$ & & Reaction & $A$ & $n$ & $E a$ \\
\hline 1 & $\begin{array}{c}\mathrm{H}_{2}+\mathrm{O}_{2}= \\
2 \mathrm{OH}\end{array}$ & $\begin{array}{c}1.70 \times \\
10^{13} \\
\end{array}$ & 0.0 & 47780 & 11 & $\begin{array}{c}\mathrm{H}+\mathrm{H}+\mathrm{H}_{2}= \\
\mathrm{H}_{2}+\mathrm{H}_{2}\end{array}$ & $\begin{array}{c}9.20 \times \\
10^{16} \\
\end{array}$ & $|-0.6|$ & 0 \\
\hline 2 & $\begin{array}{c}\mathrm{OH}+\mathrm{H}_{2}= \\
\mathrm{H}_{2} \mathrm{O}+\mathrm{H}\end{array}$ & $\begin{array}{c}1.17 \times \\
10^{9}\end{array}$ & 1.3 & 3626 & 12 & $\begin{array}{c}\mathrm{H}+\mathrm{H}+\mathrm{H}_{2} \mathrm{O}= \\
\mathrm{H}_{2}+\mathrm{H}_{2} \mathrm{O}\end{array}$ & $\begin{array}{c}6.00 \times \\
10^{19}\end{array}$ & $|-1.3|$ & 0 \\
\hline 3 & $\begin{array}{c}\mathrm{O}+\mathrm{OH}= \\
\mathrm{O}_{2}+\mathrm{H}\end{array}$ & $\begin{array}{c}4.00 \times \\
10^{14} \\
\end{array}$ & -0.5 & 0 & 13 & $\begin{array}{c}\mathrm{H}+\mathrm{OH}+\mathrm{M}= \\
\mathrm{H}_{2} \mathrm{O}+\mathrm{M}\end{array}$ & $\begin{array}{c}1.60 \times \\
10^{23} \\
\end{array}$ & $|-2.0|$ & 0 \\
\hline 4 & $\begin{array}{l}\mathrm{O}+\mathrm{H}_{2}= \\
\mathrm{OH}+\mathrm{H}\end{array}$ & $\begin{array}{c}5.06 \times \\
10^{4}\end{array}$ & 2.7 & 6290 & 14 & $\begin{array}{c}\mathrm{H}+\mathrm{O}+\mathrm{M}= \\
\mathrm{OH}+\mathrm{M}\end{array}$ & $\begin{array}{c}6.20 \times \\
10^{16} \\
\end{array}$ & $-0.6 \mid$ & 0 \\
\hline 5 & $\begin{array}{c}\mathrm{H}+\mathrm{O}_{2}+\mathrm{M}= \\
\mathrm{HO}_{2}+\mathrm{M}\end{array}$ & $\begin{array}{c}3.61 \times \\
10^{17} \\
\end{array}$ & -0.7 & 0 & 15 & $\begin{array}{c}\mathrm{O}+\mathrm{O}+\mathrm{M}= \\
\mathrm{O}_{2}+\mathrm{M}\end{array}$ & $\begin{array}{c}1.89 \times \\
10^{13} \\
\end{array}$ & 0.0 & $\mid-1788$ \\
\hline 6 & $\begin{array}{c}\mathrm{OH}+\mathrm{HO}_{2}= \\
\mathrm{H}_{2} \mathrm{O}+\mathrm{O}_{2}\end{array}$ & $\begin{array}{c}7.50 \times \\
10^{12} \\
\end{array}$ & 0.0 & 0 & 16 & $\begin{array}{c}\mathrm{H}+\mathrm{HO}_{2}= \\
\mathrm{H}_{2}+\mathrm{O}_{2}\end{array}$ & $\begin{array}{c}1.25 \times \\
10^{13} \\
\end{array}$ & 0.0 & 0 \\
\hline 7 & $\begin{array}{c}\mathrm{H}+\mathrm{HO}_{2}= \\
2 \mathrm{OH}\end{array}$ & $\begin{array}{c}1.40 \times \\
10^{14} \\
\end{array}$ & 0.0 & 1073 & 17 & $\begin{array}{c}\mathrm{HO}_{2}+\mathrm{HO}_{2}= \\
\mathrm{H}_{2} \mathrm{O}_{2}+\mathrm{O}_{2}\end{array}$ & $\begin{array}{c}2.00 \times \\
10^{12} \\
\end{array}$ & 0.0 & 0 \\
\hline 8 & $\begin{array}{c}\mathrm{O}+\mathrm{HO}_{2}= \\
\mathrm{O}_{2}+\mathrm{OH}\end{array}$ & $\begin{array}{c}1.40 \times \\
10^{13} \\
\end{array}$ & 0.0 & 1073 & 18 & $\begin{array}{c}\mathrm{H}_{2} \mathrm{O}_{2}+\mathrm{M}= \\
2 \mathrm{OH}+\mathrm{M}\end{array}$ & $\begin{array}{c}1.30 \times \\
10^{17} \\
\end{array}$ & 0.0 & 45500 \\
\hline 9 & $\begin{array}{l}2 \mathrm{OH}= \\
\mathrm{O}+\mathrm{H}_{2} \mathrm{O}\end{array}$ & $\begin{array}{c}6.00 \times \\
10^{8} \\
\end{array}$ & 1.3 & 0 & 19 & $\begin{array}{c}\mathrm{H}_{2} \mathrm{O}_{2}+\mathrm{H}= \\
\mathrm{HO}_{2}+\mathrm{H}_{2} \\
\end{array}$ & $\begin{array}{c}1.60 \times \\
10^{12} \\
\end{array}$ & 0.0 & 3800 \\
\hline 10 & $\begin{array}{c}\mathrm{H}+\mathrm{H}+\mathrm{M}= \\
\mathrm{H}_{2}+\mathrm{M}\end{array}$ & $\begin{array}{r}1.00 \times \\
10^{18} \\
\end{array}$ & -1.0 & 0 & 20 & $\begin{array}{c}\mathrm{H}_{2} \mathrm{O}_{2}+\mathrm{OH}= \\
\mathrm{H}_{2} \mathrm{O}+\mathrm{HO}_{2}\end{array}$ & $\begin{array}{c}1.00 \times \\
10^{13} \\
\end{array}$ & 0.0 & 1800 \\
\hline
\end{tabular}
drogen-oxygen mixture (unit : mole, s, cm, K, cal)

Third-body effect coefficients : (5) $\mathrm{H}_{2} \mathrm{O}=18.6, \mathrm{H}_{2}=2.86$; (10) $\mathrm{H}_{2} \mathrm{O}=0$, $\mathrm{H}_{2}=0$; (13) $\mathrm{H}_{2} \mathrm{O}=5.0$; (14) $\mathrm{H}_{2} \mathrm{O}=5.0$; others $=1.0$

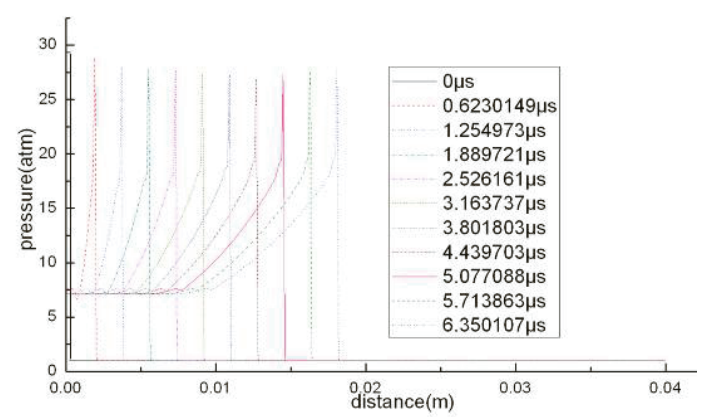

(a)

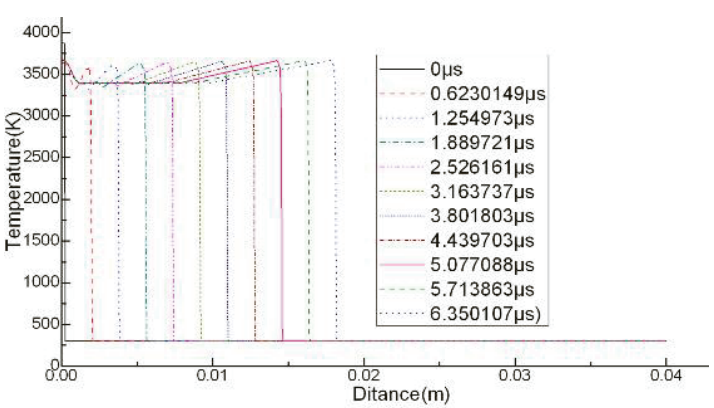

(b)

Fig. 8 Development of (a) pressure and (b) temperature

From Fig. 8, it is found that the detonation wave is very stable and detonation velocity is constant. Experimental detonation velocity is $2320 \mathrm{~m} / \mathrm{s}$. The theoretical value of the CJ model is $2333 \mathrm{~m} / \mathrm{s}$ and $2314 \mathrm{~m} / \mathrm{s}$ for our numerical result. The experimental CJ pressure of detonation is $26.1 \mathrm{~atm}, 32.43 \mathrm{~atm}$ for the theoretical value of the CJ model, and 25.66atm for our numerical result. Clearly, numerical detonation velocities and CJ pressures coincide well with the experimental and theoretical results. 


\section{CELLULAR STRUCTURE OF GASEOUS DETONATION WAVES}

When a detonation wave passed in a straight channel or reflected on a wedge, a cellular pattern in flow field can be formed. The cellular pattern is one of fundamental problems in detonation process. The generation and evolution of the cellular pattern have been studied by many scholars [12-15], but the basic mechanism of cellular pattern is still not very clear up to date. Guo et al. [9] presented a series of soot track photo pictures of cellular pattern formed by gaseous detonation waves passing in a straight channel and reflecting on a wedge. $\mathrm{Hu}$ et al. [11] have simulated cellular patterns of detonation wave.

In this study the second-order $\mathrm{CE} / \mathrm{SE}$ scheme and detailed chemical reaction model were used to numerically simulate the cellular pattern of detonation. Our cellular pattern will be compared qualitatively with experimental photo pictures obtained from Guo et al. [9].

The source items in the governing eq. (16) were used to solve the production rates of chemical reactions. In the chemical reaction flow, the gap of the characteristic time scale between chemical reaction dynamics and fluid dynamics forms the rigidity problem. In this study the decoupling method was applied to treat the rigidity problem.

In order to gain a cellular pattern, an initial perturbation must be injected into flow field. In this simulation, an initial perturbation was injected into the total specific energy or local specific energy-release at 20-50 time steps in a reaction zone. The maximum pressure in the flow field is recorded to show cellular pattern.

\subsection{Cellular Pattern of Gaseous Detonation Propa- gation Process}

Figure 9 shows numerical and experimental cellular structures. Figure 10 shows the density, pressure and temperature contours. From Figs. 9 and 10, one can see that the triple points, Mach stems, incident and transverse waves, and slip lines are simulated distinctly. The locations of slip lines between transverse waves and Mach stems can be confirmed by the discontinuity surface of density and temperature contours.

\subsection{Cellular Structure of Mach Reflection of Gase- ous Detonation on a Wedge}

Experimental and numerical cellular patterns of a Mach reflected detonation wave on a wedge are shown in Fig. 11. The cell size was changed when detonation wave passed over a wedge. Our numerical result well shows this change of cell size.

From Figs. 9 and 11, it is shown that the comparison is very satisfactory, and our second-order $\mathrm{CE} / \mathrm{SE}$ method is successful for simulating the cellular pattern of detonation wave.

\section{CONCLUSIONS}

1. The second-order CE/SE method works well for various problems of fluid dynamics, especially shock waves, vortex and interactions of shock

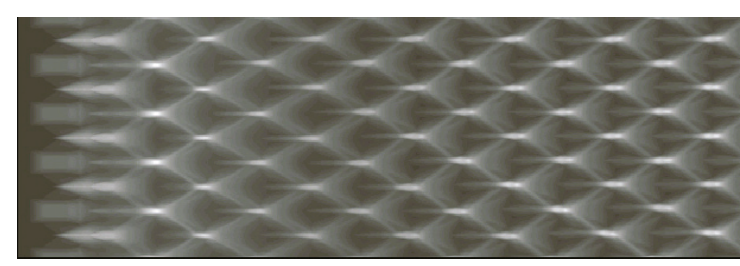

(a) Numerical result $(600 \times 200$ meshes $)$

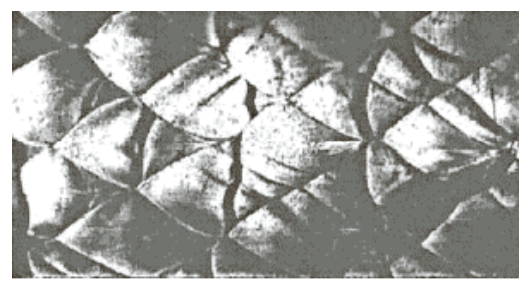

(b) Experimental result [9]

Fig. 9 Comparison of (a) the present numerical result with (b) experimental cellular pattern of detonation process

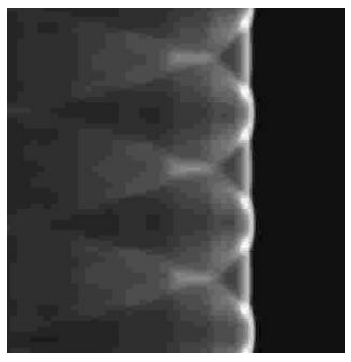

(a) Density contours

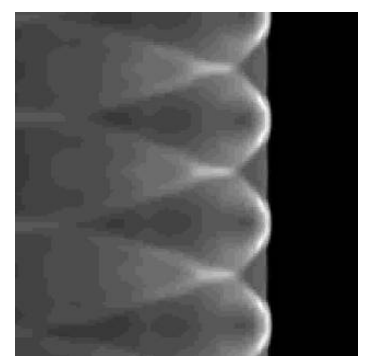

(b) Pressure contours

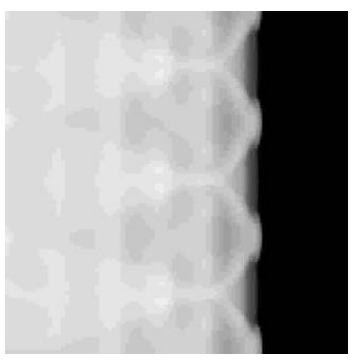

(c) Temperature contours

Fig. 10 Numerical results of (a) density, (b) pressure and (c) temperature contours

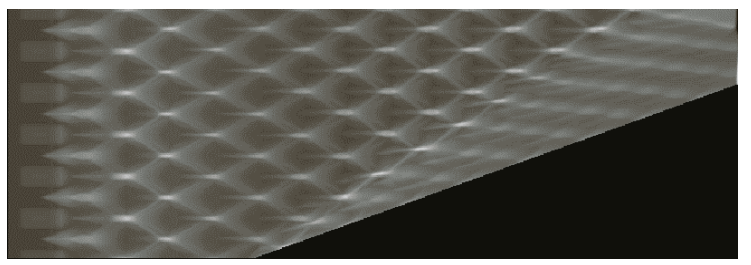

(a) Numerical result $(600 \times 200$ meshes $)$

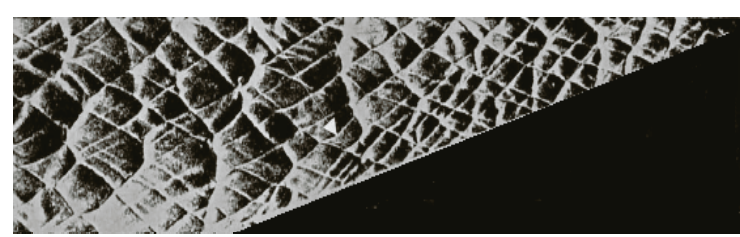

(b) Experimental result [9]

Fig. 11 Comparison of (a) numerical and (b) experimental results of cellular pattern 
wave with vortex or obstacles, and is promising for simulating complicated problems such as detonation wave propagation. Its advantages are simpler scheme, high resolution and less computational time.

2. Based on Chang's idea [1], the definitions of SE and $\mathrm{CE}$ for the original $\mathrm{CE} / \mathrm{SE}$ method have been modified and improved by a hexahedral definition. From the computational examples mentioned above, it is believed that our modification and improvement for the CE/SE method are successful.

3. With the second-order CE/SE scheme, our computational results have well compared with experiment results, exact solution and other existing computational results. It is shown that computational accuracy of the high-order CE/SE scheme is satisfactory. It was found that computational time is less and that accuracy is higher than that of the original Chang's $\mathrm{CE} / \mathrm{SE}$. Moreover, the present method seemed easier than that of a WENO scheme for capturing shock waves [16].

4. Computational results showed that the second- order $\mathrm{CE} / \mathrm{SE}$ scheme can capture a detailed structure of Mach reflections of shock wave and detonation. Especially when a detonation wave passes over a wedge, the change of cell size for cellular patterns can be successfully simulated.

\section{ACKNOWLEDGEMENTS}

The support of Far East University for D. L. Zhang during his 6-month visit was gratefully acknowledged. The authors were also indebted to reviewers' suggestions.

\section{REFERENCES}

1. Chang, S. C., "The Method of Space-Time Conservation Element and Solution Element-A New Approach for Solving the Navier-Stokes and Euler Equations," Journal of Computer Physics, 119, pp. 295-324 (1995).

2. Liu, H. T. and $\mathrm{Xu}$, J. Z., "A Space-Time Conservation Scheme for Solving the Two- Dimensional Euler Equations," Journal of Engineering Thermophysics, 18, pp. 294-299 (1997).

3. Zhang, D. L., Guo, C. M. and Xie, W., "Numerical Simulation of Cellular structure and Mach Reflection of Gaseous Detonation Wave," Explosion and Shock Waves, 21, pp. 1-7 (2001).

4. Zhang, Z. C., Yu, S. T. J. and Chang, S. C., "A SpaceTime Conservation Element and Solution Element Method for Solving Two- and Three-Dimensional Unsteady Euler Equations Using Quadrilateral and Hexahedral Meshes," Journal of Computer Physics, 175, pp. 168-199 (2002).
5. Chang, S. C., "The a(3) Scheme-A Fourth Order Neutrally Stable CE/SE Solver," 18th AIAA Computational Fluid Dynamics Conference, 25-28, Miami, FL., AIAA, pp. 2007-4321 (2007).

6. Chang, S. C., "The a(4) Scheme-A High Order Neutrally Stable CE/SE Solver," 43rd AIAA/ASME/SAE/ASEE Joint Propulsion Conference and Exhibit, 8-11, Cincinnati, OH, AIAA, pp. 2007-5820 (2007).

7. Chang, S. C., Wang, X. Y. and Chow, C. Y., "The Method of Space-Time Conservation Element and Solution Element-Applications to One- and Two- Dimensional Time-Marching Flow Problems," 12th AIAA CFD Conference, 19-22, San Diego, Ca., AIAA 95-1754-CP (1995).

8. Ben-Dor, G., "Region and Transitions of Non-stationary Oblique Shock Wave Reflection in Nitrogen and Argon: Experimental Results," UTIAS Report, 237 (1978).

9. Guo, C. M., Zhang, D. L. and Xie, W. "The Mach Reflection of a Detonation Based on Soot Track Measurements," Combustion and Flame, 127, pp. 2051-2058 (2001).

10. Zhang, D.-L., Wong, J.-T. and Liu, K., "High-order CE/SE Method and Applications," Chinese Journal of Computational Physics, 24, pp. 395-401 (2007).

11. Hu, X. Y., Zhang, D. L., Khoo, B. C. and Jiang, Z. L., "Structure and Evolution of a Two-Dimensional H2/O2/Ar Cellular Detonation," Shock Waves, 14, pp. 37-44 (2005).

12. Lee, J. H. S. and Radulescu, M. I., "On the Hydrodynamic Thickness of Cellular Detonations," Shock Waves, 41, pp. 745-765 (2005).

13. Achasov, O. V. and Penyazkov, O. G., "Dynamics Study of Detonation Wave Cellular Structure 1: Statistical Properties of Detonation Wave Front," Shock Waves, 11, pp. 297-308 (2002).

14. Lefebvre, M. H. and Oran, E. S., "Analysis of Shock Structures in a Regular Detonation," Shock Waves, 4, pp. 277-283 (1995).

15. Ohyagi, S., Obara, T., Nakata, F, and Hoshi, S., "A Numerical Simulation of Reflection Processes of a Detonation Wave on a Wedge," Shock Waves, 10, pp. 185-190 (2000).

16. Liang, S. M. and Yuan, J. C., "Numerical Simulation of Blast-Wave Propagation in a Small Two-medium Duct," Journal of Mechanics, 25, pp. 313-322 (2009).

(Manuscript received July 29, 2009, accepted for publication March 5, 2010.) 